\title{
DIGITAL TECHNOLOGY Digital safety: the next frontier for patient safety
}

\author{
Authors: Kelsey Flott, ${ }^{A}$ James Maguire ${ }^{B}$ and Natasha Phillips ${ }^{C}$
}

Healthcare safety is the product of many interrelated parts of the health system that change over time, so efforts to improve safety also need to evolve and modernise to pre-empt emerging risks. One major shift is that the records and data infrastructure necessary to support safety improvements and evaluation are increasingly facilitated by, or dependent on, digital technologies. Continuing to reduce harm and save lives requires taking a closer look at digital technologies, both in the risks they present and the solutions they offer. The priorities for digital clinical safety can be considered in two parts: first, the intrinsic safety of technologies, and second, the extrinsic ability of technologies to drive safety. Embracing these two components requires digital clinical safety to become part of the healthcare culture, with everyone understanding their role in digital clinical safety. It must include processes being easy to follow with clear, accessible guidance, accompanied by targeted standards. Patients and staff must be equipped and empowered via digital clinical safety training. Finally, the vision for digital safety includes safety solutions that should be digitally enabled, with digital technologies appropriately applied to tackle major patient safety issues.

KEYWORDS: digital, safety, patient safety

DOI: 10.7861/fhj.2021-0152

\section{Background}

Patient safety has been established as an important characteristic of quality across health systems internationally. ${ }^{1}$ It is an established academic discipline, a global government priority and an ambition of digital health innovations across the world. The Ministerial Summit for Patient Safety series galvanised support for patient safety amongst the senior echelons of health ministries, resulting in the collective prioritisation of safer care across 44 countries, and the WHO Global Patient Safety Network have accelerated the diffusion of learning for patient safety to 143 countries..$^{2-5}$ The Global Action Plan on patient safety has been upheld by the World Health Assembly, enshrining patient safety as a global priority. ${ }^{6}$

Authors: Adeputy director of patient safety, NHSX, London, UK; ${ }^{B}$ Clinical lead, NHSX, London, UK; ' Chief nursing information officer and director of patient safety, NHSX, London, UK
However, safety is a moving target and ensuring safe care requires evolution in response to new and emerging risks. ${ }^{7}$ The scope for improving patient safety remains considerable. It has been estimated that improvements in patient safety could save almost 1,000 extra lives and $£ 100$ million in care costs each year in the UK. ' Six types of events (hip fracture, death in low mortality treatment groups, pressure ulcers, sepsis, blood clots and central line infections) account for 36,000 healthy life years lost each year in England. The cost of common adverse events in England is equivalent to employing $2,000 \mathrm{GPs}$ or 3,500 hospital nurses each year. ${ }^{8}$

Safety is also the product of many interrelated parts of the health system that change over time. Safety interventions, which once focused on incident reporting and cataloguing harm, now centre on open cultures, human-centred design and innovation. One major shift is that the records and data infrastructure necessary to support safety improvements and evaluation are increasingly facilitated by, or dependent on, digital technologies.'

Taking a closer look at digital technologies, both the risks they present and the solutions they offer, is essential.

\section{The current state of digital safety}

Digital health - or the digital transformation of healthcare increasingly features as a primary ambition of health ministries and governments. In 2018, digital technologies were recognised by the WHO General Assembly as an important facilitator of all sustainable development goals, including those for Universal Health Care (UHC). ${ }^{9}$ This elevated the role of health provided by mobile phone, or mHealth, decision support and virtual approaches to capability building across low-resource health systems. ${ }^{9}$ The use of digital health technologies has become a familiar part of everyday health systems and novel digital innovation continues at pace. For instance, in England, electronic prescribing is used in over $90 \%$ of GP practices and $77 \%$ of hospital use an electronic health record (HER) system. ${ }^{10,11}$

The COVID-19 pandemic has further accelerated their use, irreversibly changing the landscape for digital health technologies. Prior to the pandemic, nearly $80 \%$ of all GP appointments in England were face-to-face, but this had fallen to just under $50 \%$ by June $2020 .{ }^{12}$ Since the start of the pandemic, $97 \%$ of UK adults have used technology to receive NHS care or to communicate with the health service. ${ }^{13}$ Technologies like the NHS App have seen downloads increase to over 12 million following the introduction of the COVID-19 passport - resulting in more than 16 million users of the NHS App. ${ }^{14}$ 
The full impact of these changes is not yet understood, and healthcare providers and policy makers are interested in measuring and sustaining the safety of these technologies. ${ }^{13}$

Across safety-critical industries, digital technologies are considered both in terms of their opportunity to enhance safety and their potential to disrupt it. There are numerous examples of healthcare safety opportunities. Pharmacist-led information technology interventions for medication errors (PINCER) has been shown to be effective in reducing medication errors, identifying 216,626 at-risk patients in at least one prescribing safety indicator (equivalent to 8.14 per 1,000 population). ${ }^{15,16}$ Scan 4 Safety has used barcodes to track data and devices, saving staff time while reducing device and identification errors. ${ }^{17}$ Remote monitoring technologies are demonstrating an ability to recognise early signs of deterioration. ${ }^{18}$

From a disruption perspective, however, a growing evidence base also highlights the risks attached to new digital technologies. New technologies parachuted into organisations can increase cognitive burden for staff, exacerbating the risk of error. ${ }^{19}$ Oversights in user-centred design and unanticipated malfunctions can result in unintentional error that is both costly and damaging. ${ }^{19}$ Evidence from England suggests that incidents involving digital technologies are preventable in $75 \%$ of cases. ${ }^{20}$

\section{Ensuring the intrinsic safety of digital technologies}

The first priority for digital clinical safety, as in all aspects of healthcare, is 'first, do no harm.' However, the standards, guidance and training that underpin the safe use of digital technologies are less commonly discussed in patient safety than might be appropriate for modern healthcare.

\section{Standards and guidance}

The authority on whether or not a digital health technology is safe usually rests in a law, upheld by local or national regulation. In England, clinical risk management standards DCB0129 for manufacturers and DCB 0160 for deploying organisations exist to ensure that both suppliers and users of digital technologies provide an adequately risk-mitigated safety case. ${ }^{21,22}$ The Health and Care Act of 2012 stipulates the requirement for these standards. ${ }^{21,22}$ These sit alongside other medical device legislation, but are not always known or understood to users, commissioners and developers of digital health technologies.

Clinical risk management standards are one set of requirements in a network of regulations including cyber security and Information Governance. The digital technology assessment criteria (DTAC) provide a coherent overview of all assessment criteria for digital systems and aim to ensure these requirements align and support one another rather than placing an insurmountable barrier to entry for technology developers. ${ }^{23}$

Standards and guidance are a foundation of a safety culture, but can be a challenge to implement reliably. Articulating key messages from standards and guidance simply, as well as showcasing best practice, could facilitate a more user-friendly interaction with this component of digital clinical safety. A central repository to understand how to optimise commonly used digital technologies, or clear blueprints for how best to conduct ensure digital clinical safety, could facilitate broader engagement with standards and improve safety. Furthermore, as current standards are reviewed and new ones are created, it will be important to use principles of co-production to ensure standards are written in a patient-centric way. Despite challenges to effective co-production, extending participation through open consultations can still support a diversity of input into standard creation.

\section{Training and education}

Healthcare staff and suppliers of technologies need to be equipped to meet the standards and guidance. Risk mitigation for hazards across different scenarios of a product's use takes both clinical and technological expertise. In the UK, existing training provided by NHS Digital supports manufacturers and implementers of technologies to understand the risk mitigation activities required to effective and continuously uphold the clinical safety standards. ${ }^{24}$

However, more broad-spectrum training for digital clinical safety will help embed the concepts of digital safety across health systems. Components of traditional patient safety and digital health education share common elements from disciplines including human factors, innovation and healthcare leadership. Given this crossover and the existing foundations for patient safety training, including the NHS Patient Safety Syllabus, there is a window of opportunity to bridge the curricula and ensure digital clinical safety is integrated in patient safety training and vice versa. ${ }^{25}$

Ensuring digital technologies are safe is often a process that happens behind the scenes and is not captured in grey or academic literature. Individual examples of projects that have ensured the safety of digital technologies help to demonstrate this element of digital safety (see Box 1).

Programmes that not only adhere to existing guidance, but help to build capacity and expertise across the workforce, support a concerted effort to ensure the safety of technologies. They demonstrate the need not only to comply with guidance, but to capture and share examples of how best to ensure the safety of digital technologies.

\section{Using digital technologies to improve safety}

\section{Building on success}

As evidenced above, certain aspects of healthcare have already been transformed by digital technologies with proven benefits to safety. Where digital technologies have improved safety, they have been designed with the needs of users in mind and relevant training and support has been provided. These examples serve as a blueprint for how digital technologies can safely and effectively

\section{Box 1. Case study: Ensuring the safety of digital} technologies -the electronic eye referral systems programme 26

The NHSX electronic eye care referral systems (EeRS) programme has introduced digital referral solutions to streamline communication between eye care providers and hospitals. Throughout the programme's development and delivery, information about the clinical safety standards and other relevant processes were cascaded to frontline delivery partners at regular meetings and clinical safety clinics were organised to help staff to resolve issues and to facilitate the sharing of supporting guidance. These clinics covered how to complete the documentation for safety case reports, how to ensure effective risk mitigation and control and how to work with others to share information about clinical safety risk management. 
improve health services, both solving a problem and disrupting a status quo without undue risk. They help to dispel the myth of a trade-off between safety and innovation.

\section{Ensuring involvement and inclusion}

Proactively applying digital technologies to major safety priorities requires some assurance that technologies meet the needs of patients, frontline staff and citizens.

This requires consideration about the role digital technologies have in both widening and narrowing existing health inequalities. The recent acceleration in the use of digital technologies has raised questions about the future of their adoption and scale as safety solutions. The pandemic has laid bare the problem of digital exclusion, where certain people or groups have continuing unequal access and capacity to use essential information and communications technologies ${ }^{27,28}$ - a reality which extends to approximately $22 \%$ of the UK population. ${ }^{29}$ However, it has also demonstrated the power of digital technologies to connect people to health services in the most challenging of times. Technologies like remote monitoring have the power to address major safety issues around early detection of deterioration outside of hospital.

Digital clinical safety must extend to everyone, and anticipating these challenges and pre-empting them will be key to avoid exacerbating problems associated with digital exclusion. This will require active co-production and partnership between citizens, the professional healthcare community and innovators. While digital safety will not solve the systemic problems of digital exclusion, a concerted effort will mitigate the risk of technology exacerbating it and maximise the benefits.

\section{Identifying opportunities for innovation}

Surfacing opportunities for digital technologies to drive safer care also requires a rigorous and data-driven strategy. Applying digital interventions to enduring safety challenges is a priority requiring careful evidence-based consideration. While evidence already suggests that digital improvements to existing technologies can reduce harm, ${ }^{30}$ more research is needed to indicate where digital solutions would be most appropriate and how these technologies can be specifically designed and implemented within a health system. This requires collaborative effort across all sectors to ensure patient safety expertise and digital health expertise are represented in crafting solutions.

At the highest level, digital clinical safety also requires stronger prioritisation and active discourse at the policy making and regulatory level. Digital can help us capitalise on enthusiasm for improvement in patient safety, but only if we get the foundations and the collaborations right first.

The Scan4Safety programme is a real-world example of the potential digital technologies have to improve patient safety (Box 2). The use of scanning and tracking technologies will continue to expand through national projects including the Medical Device Safety Programme, and clearly demonstrates how technologies can yield a measurable safety benefit.

\section{A vision for the future}

Moving towards safer digital health systems requires a clear strategy and a shared vision. While this vision is still developing, key principles should underpin it to maximise digital clinical safety. We recommend five key principles (see Box 3).
Box 2. Case study: Using digital technologies to improve safety - Scan for Safety ${ }^{31}$

The programme operates on the evidence-based premise that scanning and barcode tracking technologies can support the reduction of error and collection of data. Implementing scanning and tracking protocols consistently across a number of NHS hospitals demonstrated a reduction in patient identification errors and never events, whilst also improving traceability of devices and facilitating product recalls when required. One pharmacy department in the pilot found the technologies reduced error rates by up to $76 \% .{ }^{17}$ Furthermore, in Scan 4 Safety demonstrator sites, scanning technologies proved beneficial in terms of improving time released for patient care equivalent to 16 band 5 nurses per organisation as well as improved operational costs with $£ 2.75 \mathrm{~m}$ efficiency savings per organisation annually.

Digital clinical safety should become part of the healthcare culture and system, with everyone understands their role in this area. This includes clear national and local governance structures. Processes should be easy to follow with clear, accessible and targeted standards and guidance. Individuals should be equipped and empowered through digital clinical safety training that is expanded and promoted across local health and care systems. Safety solutions should be digitally enabled, with digital technologies appropriately applied to tackle major patient safety issues. Safety decisions must be data-driven, meaning digital clinical safety data is captured, reviewed and used to drive improvements.

\section{Conclusion}

Patient safety as a discipline and as a global health priority has gathered momentum in health policy over the past two decades. With a push from the pandemic, digital safety has also landed in the limelight as a necessary and critical component of sustaining the positive legacy of digital technologies in healthcare.

Patient safety is now inextricably linked to digital transformation and the promise of a safe health system for the future rests in

\section{Box 3. Principles for digital clinical safety}

Collaboration: Partnership working between health and care organisations, regulators, industry, the scientific community and patients and citizens to ensure risks are understood and managed effectively.

> Openness: Sharing of important information on potential risks to ensure appropriate escalation and mitigation activity.

> Iterative change: Identifying learning from events to ensure systems are safe and using feedback loops wherever possible.

> Proportionality: Developing processes that are proportionate to the scale, complexity and level of clinical risk associated with the programme of work. Proportionality ensures that risks are mitigated and controlled in accordance with their likelihood and severity.

Inclusion: Encouraging active co-production in digital safety processes to put patients, staff and the public at the heart of digital safety. 
digital clinical safety. For this to materialise, we need a strategy and standards, but we also need rounded education, a culture of sharing best-practice and an appetite for collaboration and innovation. The vision for a safe digital health system is one that can be achieved, but only if it is prioritised across health systems, industry partners and citizens.

\section{Acknowledgements}

We wish to thank the ongoing support from the NHS Digital Clinical Safety team, the NHS England and NHS Improvement Patient Safety team as well as from teams across NHSX.

\section{References}

1 National Academies of Sciences, Engineering, and Medicine. Crossing the global quality chasm: improving health care worldwide. Washington, DC: The National Academies Press, 2018.

2 Flott K, Durkin M, Darzi A. The Tokyo Declaration on patient safety. BMJ 2018;362:k3424.

3 Nicholson D, Yates R, Warburton W, Fontana G. Delivering universal health coverage - a guide for policymakers. World Innovation Summit for Health, 2015. Available from https://www. wish.org.qa/wp-content/uploads/2018/01/WISH_UHC_Forum_ Report_08.01.15_WEB.pdf [Accessed 21 Oct 2021].

4 Flott K, Fontana G, Dhingra-Kumar N, et al. Health care must mean safe care: enshrining patient safety in global health. Lancet 2017;389:1279-81.

5 WHO Global Patient Safety Network. WHO Global Patient Safety Network. https://ezcollab.who.int/gpsn/ [Accessed 21 Oct 2021].

6 World Health Organization. Global patient safety action plan 2021-2030: towards eliminating avoidable harm in health care. WHO, 2021.

7 NHS England and NHS Improvement. The NHS patient safety strategy. www.england.nhs.uk/patient-safety/the-nhs-patient-safety-strategy/\#patient-safety-strategy [Accessed 21 Oct 2021].

8 Slawomirski L, Auraaen A, Klazinga K. The economics of patient safety. Organisation for Economic Co-operation and Development, 2017. www.oecd.org/health/health-systems/The-economics-ofpatient-safety-March-2017.pdf [Accessed 21 Oct 2021].

9 Labrique A, Vasudevan L, Mehl G, Rosskam E, Hyder AA. Digital health and health systems of the future. Glob Health Sci Pract 2018;6:S1-S4.

10 Warren LR, Clarke J, Arora $S$ et al. Improving data sharing between acute hospitals in England: an overview of health record system distribution and retrospective observational analysis of interhospital transitions of care. BMJ Open 2019;9:e031637.

11 NHS England and NHS Improvement. The Long Term Plan. Chapter 5: Digitally-enabled care will go mainstream across the NHS. www. longtermplan.nhs.uk/online-version/chapter-5-digitally-enabledcare-will-go-mainstream-across-the-nhs/ [Accessed 21 Oct 2021].

12 Horton T, Hardie T, Mahadeva S, Warburton W. Securing a positive health care technology legacy from COVID-19. Health Foundation, 2021. www. health.org.uk/publications/long-reads/securing-a-positive-health-caretechnology-legacy-from-covid-19 [Accessed 21 Oct 2021].

13 Spencer J, Oung C. How has lockdown affected general practice and where do we go from here? Nuffield Trust, 2021. www.nuffieldtrust.org.uk/news-item/how-has-lockdown-affected-generalpractice-and-where-do-we-go-from-here [Accessed 21 Oct 2021].

14 Downey A. NHS App hits milestone $12 \mathrm{~m}$ downloads since Covid passports introduced. DigitalHealth, 2021. www.digitalhealth. net/2021/09/nhs-app-hits-milestone-12m-downloads-since-covidpassports-introduced/ [Accessed 21 Oct 2021].

15 Avery A, Rodger S, Cantrill J et al. A pharmacist-led information technology intervention for medication errors (PINCER): a multicentre, cluster randomised, controlled trial and cost-effectiveness analysis. Lancet 2012;379:1310-19.
16 University of Nottingham. PRIMIS and PINCER. www.nottingham. ac.uk/primis/pincer/pincer-intervention.aspx [Accessed 21 Oct 2021].

17 GS1 UK. A scan of the benefits: the Scan4Safety evidence report. GS1 UK, 2020. https://healthcare.gs1uk.org/scan4safety/gs1_ uk_a_scan_of_the_benefits_report.pdf [Accessed 21 Oct 2021].

18 Clarke J, Flott F, Crespo R et al. Assessing the safety of home oximetry for COVID-19: a multisite retrospective observational study. BMJ Open 2021;11:e049235.

19 Yu A, Flott K, Chainani C, Fontana G, Darz A. Patient Safety 2030 NIHR Imperial Patient Safety Translational Research Centre, 2016. www.imperial.ac.uk/media/imperial-college/institute-of-globalhealth-innovation/centre-for-health-policy/Patient-Safety-2030Report-VFinal.pdf [Accessed 21 Oct 2021].

20 Martin G, Ghafur S, Cingolani I et al. The effects and preventability of 2627 patient safety incidents related to health information technology failures: a retrospective analysis of 10 years of incident reporting in England and Wales. Lancet Digit Health 2019;1:E127E135.

21 NHS Digital. DCB0129: Clinical risk management: its application in the manufacture of health IT systems. 2021. https://digital.nhs.uk/ data-and-information/information-standards/informationstandards-and-data-collections-including-extractions/publicationsand-notifications/standards-and-collections/dcb0129-clinicalrisk-management-its-application-in-the-manufacture-of-health-itsystems [Accessed 21 Oct 2021].

22 NHS Digital. DCB0160: Clinical risk management: its application in the deployment and use of health IT systems. https://digital.nhs. uk/data-and-information/information-standards/informationstandards-and-data-collections-including-extractions/publicationsand-notifications/standards-and-collections/dcb0160-clinicalrisk-management-its-application-in-the-deployment-and-use-ofhealth-it-systems [Accessed 21 Oct 2021].

23 NHSX. Digital technology assessment criteria (DTAC). www.nhsx. nhs.uk/key-tools-and-info/digital-technology-assessment-criteriadtac/ [Accessed 21 Oct 2021].

24 NHS Digital. Clinical risk management training. https://digital. nhs.uk/services/clinical-safety/clinical-risk-management-training [Accessed 21 Oct 2021].

25 Health Education England. Patient safety. www.hee.nhs.uk/ourwork/patient-safety [Accessed 21 Oct 2021].

26 NHSX. Improve referrals to eye care services. www.nhsx.nhs.uk/keytools-and-info/digital-playbooks/eye-care-digital-playbook/improvereferrals-to-eye-care-services/ [Accessed 21 Oct 2021].

27 Schejter A, Ben-Harush O, Tirosh N. Re-theorizing the 'digital divide': Identifying dimensions of social exclusion in contemporary media technologies. https://eprints.qut.edu.au/86701/1/86701.pdf [Accessed 21 Oct 2021].

28 Warren M. The digital vicious cycle: Links between social disadvantage and digital exclusion in rural areas. Telecomms Policy 2007:31:374-88.

29 Lloyds Bank. UK Consumer Digital Index 2019. Lloyds Bank, 2019. www.lloydsbank.com/assets/media/pdfs/banking_with_us/ whats-happening/lb-consumer-digital-index-2019-report.pdf [Accessed 21 Oct 2021].

30 Avery AJ, Sheehan C, Bell B et al. Incidence, nature and causes of avoidable significant harm in primary care in England: retrospective case note review. BMJ Qual Saf 2020, in press (doi: 10.1136/ bmjqs-2020-011405).

31 Royal Cornwall Hospitals NHS Trust. Scan4Safety. www. royalcornwall.nhs.uk/cornwall-it-services-cits/apps-devices/ scan 4safety/ [Accessed 21 Oct 2021].

Address for correspondence: Dr Kelsey Flott, NHSX, Skipton House, 80 London Road, London SE1 6LH, UK.

Email: kelsey.flott@nhsx.nhs.uk

Twitter: @kelseyflott 